\title{
双环和多环四唑含能化合物的合成研究进展
}

\author{
彭 蕾 $a$ 李玉川 ${ }^{a}$ 杨雨璋 ${ }^{a}$ 刘 威 ${ }^{b}$ 张雪娇 ${ }^{a}$ 庞思平*, $a$ \\ $\left({ }^{a}\right.$ 北京理工大学材料学院 北京 100081) \\ $\left({ }^{b}\right.$ 北京理工大学生命学院 北京 100081)
}

\begin{abstract}
摘要 综述了近年来双环和多环四唑含能化合物的合成研究进展, 包括偶氮四唑、联四唑、双环四唑胺、肼基四唑、 硝胺基四唑、硝基四唑、烷基相连的双环四㟇、多环四㟇等，结合化合物的表征，对双环和多环四㟇含能化合物的性 质做了阐述, 对双环和多环四唑含能化合物的发展做了展望.
\end{abstract}

关键字 四㟇; 含能化合物; 合成; 表征; 综述

\section{Research Progress in Synthesis of Energetic Compounds of Bicyclo- and Multicyclo-tetrazoles}

\author{
Peng, Lei ${ }^{a} \quad$ Li, Yuchuan $^{a} \quad$ Yang, Yuzhang $^{a} \quad$ Liu, Wei $^{b} \quad$ Zhang, Xuejiao ${ }^{a}$ \\ Pang, Siping ${ }^{*, a}$ \\ ( ${ }^{a}$ School of Materials Science and Engineering, Beijing Institute of Technology, Beijing 100081) \\ ( ${ }^{b}$ School of Science and Technology, Beijing Institute of Technology, Beijing 100081)
}

\begin{abstract}
The progress in synthesis of bicyclotetrazoles and multicylcotetrazoles energetic compounds is reviewed, including azotetrazoles, bistetrazoles, bis(tetrazole)amine, hydrazinebistetrazoles, nitramino-bicycoltetrazoles, nitro-bicyclotetrazoles, alkyl-bicyclotetrazole, multicyclotetrazoles, elaborating properties of bicyclotetrazoles and multicyclotetrazoles from the characterization. And the progress of bicyclotetrazoles and multicyclotetrazoles energetic compounds in the future is also discussed.

Keywords tetreazole; energetic compounds; synthesis; characterization; review
\end{abstract}

四唑环氮含量超过 $80.0 \%$, 是目前能够稳定存在的 含氮量最高的一种环结构单元. 四㭫化合物特别是双环 和多环四唑衍生物，因其含有大量高能的 $\mathrm{N}-\mathrm{N}$ 键、 $\mathrm{C}-\mathrm{N}$ 键以及大的环张力, 并且具有高密度、高生成 焓 ${ }^{[1,2] 、}$ 高气体生成量、低感度、热稳定性好以及爆轰产 物多为洁净的 $\mathrm{N}_{2}$ 等一系列优良性质, 而成为理想的高 能量密度材料(HEDMs), 获得了国内外学者的广泛关 注 $^{[3 \sim 5]}$.

近年来四唑化合物得到很大发展, 在药物合成、炸 药、推进剂等方面的应用报道较多. 四唑环一般是通过 叠氮化合物与有机氰化物进行 [3+2]环加成反应制得, Himo 等 ${ }^{[6]}$ 提出了 $\mathrm{Zn}(\mathrm{II})$ 催化有机氰化物与叠氮化物反应 生成相应四唑化合物的反应机理. 目前已经有很多关于 单环四唑类化合物的综述 ${ }^{[7]}$.
随着研究的深入, 在单环四唑化合物的基础上衍生 出双环和多环四唑化合物. 双环和多环四唑化合物比单 环四唑化合物有更多的修饰位点, 可以更好地根据需要 调节性能, 目前已合成出大量性能优越的双环和多环四 唑含能化合物, 但迄今还没有关于双环和多环四唑含能 化合物的全面综述. 笔者查阅了大量的文献，对于近年 来国内外双环和多环四唑化合物的研究包括双环及多 环四唑化合物的合成近况、表征方法、应用性能等都进 行了综述.

\section{1 双环四唑}

双环四唑化合物(bicyclotetrazoles)即为含有两个四 唑环的化合物，根据连接方式的不同，双环四唑化合物 可以分为偶氮四唑、联四唑、联氨四唑、硝胺基四唑、

\footnotetext{
* E-mail: pangsp@bit.edu.cn

Received September 6, 2011; revised November 3, 2011; published online November 21, 2011.

Project supported by the Program for New Century Excellent Talents in University (No. NCET-09-0046).

新世纪优秀人才支持计划(No. NCET-09-0046)资助项目.
} 
四唑盐等.

\section{1 偶氮四唑}

偶氮四唑含有 2 个 $\pi$ 体系, 将偶氮基引入四唑分子 结构中，既提高了化合物的氮含量又增加了其生成焓.

早在 20 世纪前, Thiele 等 ${ }^{[8]}$ 就完成了 5,5'-偶氮四唑 (1)的合成和表征, 晶体结构测试证实在一 $30{ }^{\circ} \mathrm{C}$ 以下 1 与甲醇形成加合物, 并且只在固态时才能稳定存在, 在 室温条件下该物质很容易分解为 5-肼基- $1 H$-四唑和甲 酸.<smiles>N(=Nc1nnn[nH]1)c1nnn[nH]1</smiles>

由于稳定性差， 1 在实际应用中受限，但是高氮含 量 $(85.4 \%)$ 使其成为一个令人着迷的高能化合物结构单 元, 1 的阴离子可以与别的基团组成离子化合物(含能离 子盐 ${ }^{[9 \sim 11]}$, 初步实验结果显示, 其可望作为调整火箭推 进剂燃烧速率的材料、气囊的气体发生剂、炸药等使用.

\subsection{1 烷基取代的偶氮四唑化合物}

2010 年 Serebryanskaya 等 ${ }^{[12]}$ 报道了烷基取代的 5氨基四唑(5-AT)氧化偶联为烷基偶氮四唑 $\mathbf{2}$ 和 $\mathbf{3}$ 的新方 法(Scheme 1). 该方法采用廉价易得的 $N$-溴代丁二酰亚 胺(NBS)为氧化剂, $\mathrm{CH}_{2} \mathrm{Cl}_{2}$ 或者 $\mathrm{CH}_{3} \mathrm{CN}$ 回流, 5-AT 衍生 物：NBS：偶氮二异丁腈 $=1: 2: 0.001$ (物质的量的 比), 产率较高. X射线单晶衍射发现叔丁基取代的 5-AT 形成的偶氮四唑化合物为 $S$-cis-S-cis 结构, 而甲基取代 的 5-AT 形成的偶氮四唑化合物为 S-trans-S-trans 结构.

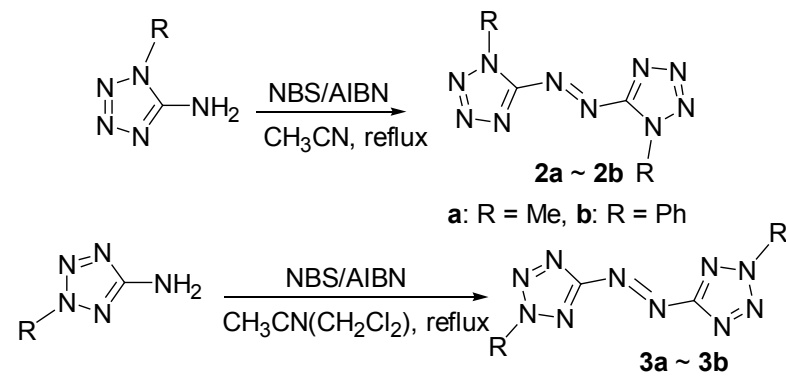

$\mathbf{a}: \mathrm{R}=\mathrm{Me}, \mathrm{b}: \mathrm{R}=t-\mathrm{Bu}$

\section{Scheme 1}

\subsection{2 偶氮四唑金属盐}

偶氮三唑、四唑金属盐可以作为高能材料 ${ }^{[13,14]}$ 或者 氮气发生剂 ${ }^{[15]} .1892$ 年 Thiele 等 ${ }^{[8]}$ 首次通过高锰酸钾氧 化 5-AT 合成出了 5,5'-偶氮四唑钠盐 4. 2002 年 Hammerl 等 ${ }^{[16]}$ 用合成出的 4 与二价氯盐反应得 5 . 偶氮四唑钡盐 $5 b$ 分别与一价、二价和三价金属硫酸盐反应得到 6,7 和 8 (Scheme 2). 偶氮四唑金属盐的水合物因为水分子 的存在感度不是很高, 一旦失水, 金属盐的感度会迅速
升高.
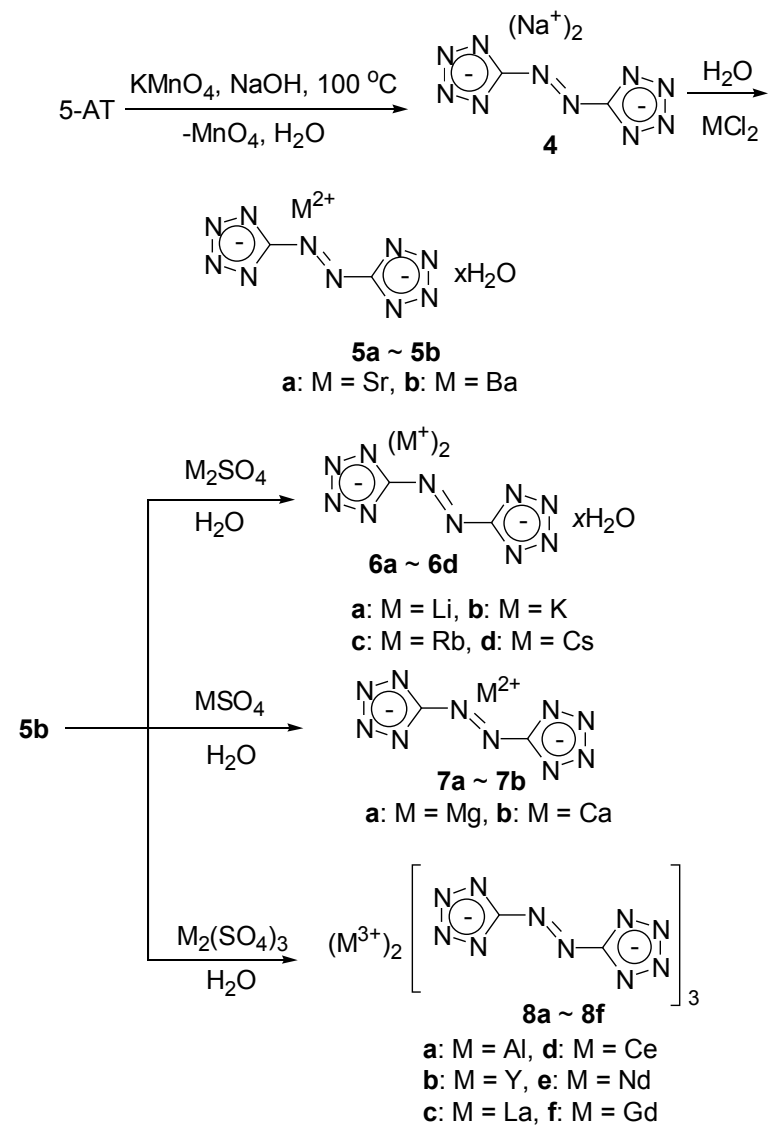

Scheme 2

化合物 4 经常结合 5 个单位的水分子形成五水合偶 氮四唑钠盐(SAZ), 该化合物是合成很多金属盐的重要 前体. 2005 年, Singh 等 ${ }^{[17]}$ 发表了 SAZ 的 UV 和 FT-IR 的 光谱数据, 其实验得到的元素分析数据与理论值误差小 于 $\pm 1 \%$

2007 年 Klapötke 等 ${ }^{[18]}$ 报道了合成偶氮四唑盐的新 方法, 他们研究发现白色固体联氨四唑(HBT, 9)可以转 化为黄色的 1 , 因此他们由 5-氨基- $1 H$-四唑经 2 步反应 以较高产率得到 $\mathbf{9}$, 然后由中间产物 9 制得对应的盐 $\mathbf{1 0}$ (Scheme 3), 用钝感的 9 替代易爆的偶氮四唑钡盐合成 其它偶氮四唑金属盐大大提高了安全性.

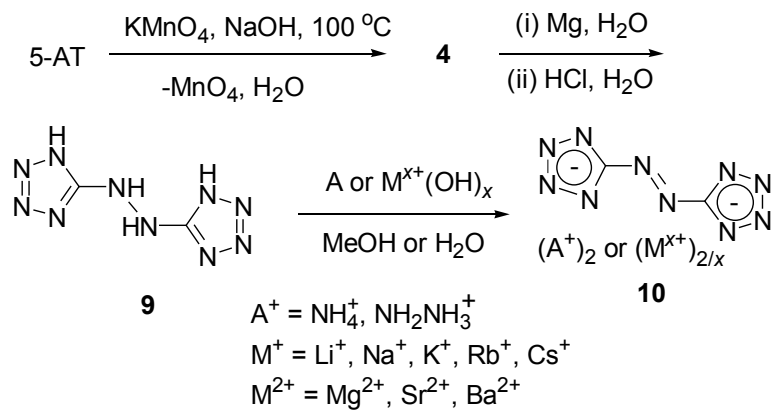

Scheme 3 
与 $\mathrm{Ba}(\mathrm{II}), \mathrm{Pb}(\mathrm{II})$ 和 $\mathrm{Hg}(\mathrm{II})$ 相比, $\mathrm{Cu}(\mathrm{II})$ 阳离子毒性较 小. 2009 年 Tao 等 ${ }^{[19]}$ 用 SAZ 分别与硫酸铜和氯化镉反应 得到相应的铜盐和镉盐的二水合物 $\mathbf{1 2}$ 和 14 (Scheme 4), 并对其进行表征, 但是这些盐的感度都较高, 且分解产 物含有的重金属对环境有害.

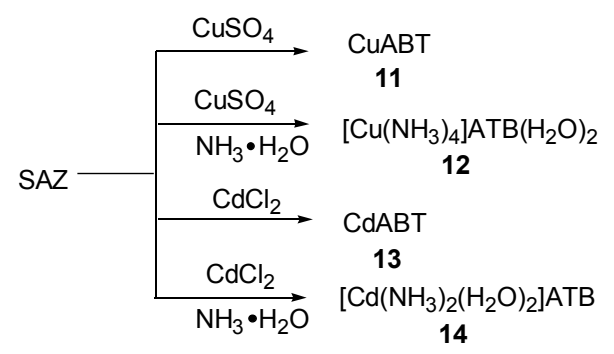

Scheme 4

\subsection{3 偶氮四唑非金属盐}

大多数偶氮四唑非金属盐与其金属盐相比, 感度降 低且不吸湿, 这些非金属盐包括铵盐、胍盐、肼盐、氨 基胍盐、二氨基胍盐、三氨基胍盐等. 氮含量从 $78.8 \%$ 的二胍盐到 $85.2 \%$ 的双腙盐衍生物, 感度都比较低, 热 稳定性可以达到 $170{ }^{\circ} \mathrm{C}$ 以上, 与 TNT 有很多相似之处. 其中偶氮四唑铵盐(AZT)不含结晶水也不吸湿, 撞击感 度与 RDX 相当; 偶氮四唑胍盐(GZT)热稳定性好, 对撞 击高度钝感; 偶氮四唑三氨基胍盐(TAGZT)生成焓高达 $1074 \mathrm{~kJ} / \mathrm{mol}$, 标准状况下单位质量成气量高达 981 $\mathrm{mL} / \mathrm{g}^{[20]}$. GZT 感度与标准的钝感炸药 TATB 相当, 生成 的气体温度低, 可代替叠氮钠应用于安全气囊装置, 以 避免叠氮钠存在的毒性以及反应时放出难闻气体等问 题. AZT 和 TAGZT 还可作为替代 HMX 的组分应用于固 体推进剂.

\subsubsection{1 偶氮四唑肼盐}

Hammerl 等 ${ }^{[1]} 2001$ 年合成出了一个新型的富氮含 能材料一偶氮四唑肼盐(10a), 氮含量超过 $85 \%$. 硫酸肼 与偶氮四唑钡盐反应得到偶氮四唑肼盐水合物, 加热失 水得到 10a (Scheme 5). Sivabalan 等 ${ }^{[21]}$ 经过分析测试提 出 $10 \mathrm{a}$ 的分解机理为: 1 分子的 $10 \mathrm{a}$ 分解得到 1 分子氢

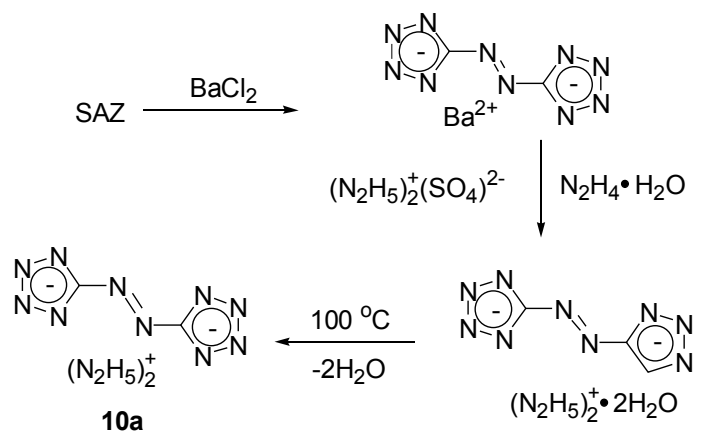

气、2 分子氮气和 3 分子甲烷. 10a 能量主要是来自高的 正生成热, 其计算密度为 $1.564 \mathrm{~g} / \mathrm{cm}^{3}$, 分解热 3743 $\mathrm{kJ} / \mathrm{mol}$, 爆炸温度为 $3200 \mathrm{~K}$, 气体生成量为 $1436 \mathrm{~L} / \mathrm{kg}$, 有望成为新型气体发生剂、低特征信号推进剂、低烟或 者无烟烟火以及高性能炸药的重要组成部分.

\subsubsection{2 偶氮四唑胍盐}

2008 年王义惠等 ${ }^{[22]}$ 报道了偶氮四唑二胍盐(GZT, 15)的合成优化与性能表征. 优化后的条件为: $\mathrm{NaOH}$ 溶 液的浓度为 $1.5 \mathrm{~mol} / \mathrm{L}, 5-\mathrm{AT}$ 与 $\mathrm{KMnO}_{4}$ 的物质的量的比 为 $3: 3$, 在 $80{ }^{\circ} \mathrm{C}$ 下反应 $60 \mathrm{~min}$, 平均产率超过 $80 \%$, 可重复性良好. 15 分子式为 $\mathrm{C}_{4} \mathrm{H}_{12} \mathrm{~N}_{16}$, 含氮量高达 $78.8 \%$, 产气量大、热稳定好、对摩擦和撞击钝感. TG-DTA 可以看出在 $251.99 \sim 253.29{ }^{\circ} \mathrm{C}$ 之间失去 $79.8 \%$ 的质量, 说明 15 分解速度很快, 是一种性能良好的新型 产气剂.

偶氮四唑三氨基胍(17)首先被印度总部的海军表面 武器中心(NSWC)研制成功. 17 是一种亮黄色针状晶体, 密度最大为 $1.60 \mathrm{~g} / \mathrm{cm}^{3}$, 分解焓为 $+1076 \mathrm{~kJ} / \mathrm{mol}$. 徐松 林等 ${ }^{[23]}$ 以 5-AT 为起始原料在温和的条件下经两步反应 合成了 17 , 产物为很纯的晶体, 产率为 $73.3 \%$, 熔点为 196 $\sim 197{ }^{\circ} \mathrm{C}$, 并对其结构进行 ${ }^{1} \mathrm{H}$ NMR, ${ }^{13} \mathrm{C}$ NMR, FT-IR 和元素分析表征. 17 可以通过 Scheme 6 所示的合 成步骤大幅度地降低生产成本, 在爆炸、烟火、气体发 生剂和低特征推进剂领域得到广泛应用. 2006年 Tappan 等 ${ }^{[24]}$ 详细报道了 17 的燃烧速率、撞击感度、摩擦感度、 热分解、燃烧速率指数等作为含能材料的基本性能指标. Tremblay ${ }^{[25]}$ 和 Hiskey 等 ${ }^{[26]}$ 先后对 17 的物理性质进行了 综合分析, 测定了 17 的燃烧速度、激光引燃和瞬间分解 特性, 通过对比实验验证: 17 与 BTATz, DAATO 和 HMX 中燃烧速率最快的 DAATO 的燃烧速率相近, 是 在低压条件下燃烧速率最快的物质之一; 17 的燃烧速率

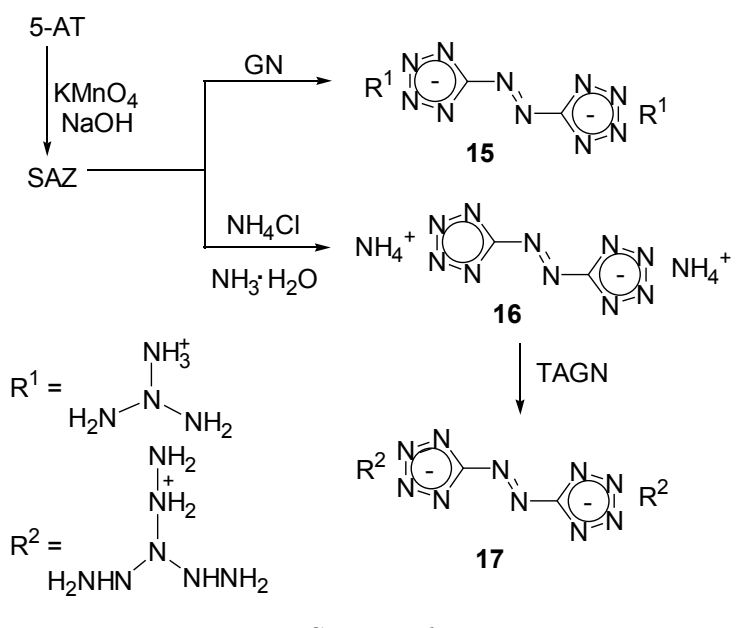

Scheme 6

Scheme 5 
的压力指数为 0.672 , 介于 $\mathrm{HMX}$ 和一些其他的高能材料 之间; 凝固相反应发出大约 $65 \%$ 的热量, 有助于其在低 压条件下达到高的燃烧速度.

\subsubsection{3 其它偶氮四唑非金属盐}

2005 年 $\mathrm{Ye}$ 等 ${ }^{[27]}$ 报道以富氮的氨基三唑、四唑等作 为阳离子与偶氮四唑钠盐和钡盐进行反应合成含能离 子盐(Scheme 7). 化合物 $18 \mathrm{a}$ 和 $18 \mathrm{~b}$ 表现出了很高的正 生成焓, 分别为 4360 和 $4679 \mathrm{~kJ} / \mathrm{mol}, \mathbf{1 8 a}, 18 b$ 和 19 的密 度为 $1.5 \sim 1.6 \mathrm{~g} / \mathrm{cm}^{3}$, 可以作为炸药、气体发生剂等应用, 并已经尝试将其用于纳米金刚石的合成.

$$
\text { (1) } \mathrm{Ag}_{2} \mathrm{SO}_{2} \mathrm{~N}_{10}
$$

Scheme 7
Klapötke 等 ${ }^{[28]}$ 以三取代四唑作为阳离子，偶氮四唑 为阴离子在温和的条件下较高产率得到相应含能离子 盐(Scheme 8), 并对这些新型化合物的光谱特性、固态 晶体结构、氢键等进行了分析表征. 所有的化合物都对 撞击、摩擦钝感, 热力学稳定性高达 $170{ }^{\circ} \mathrm{C}$ 以上. 与金 属偶氮四唑相比，此类非金属偶氮四唑的爆炸产物对环 境更友好, 但是成本较高, 目前在实际中的应用很少.

\section{2 联四唑}

1920 年首次报道用叠氮酸水溶液与氰气反应制备

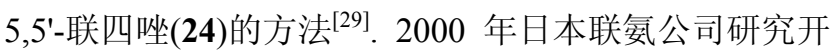
发了批量生产联四唑的工艺，采用合成联四唑铵盐的方 法来生产 24 , 将产率提高到 $70 \%$ 以上 ${ }^{[30]}$. 近期联氨公司 又采用新的合成方法，经中间产物均二肼基乙二亚胺， 联四唑铵盐产率提高到 $80 \%$ 以上，更适合工业化大规模 生产, 同时得到高纯度的联四唑铵盐 25 (Scheme 9).

Hiskey 等 ${ }^{[31]}$ 用 24 分别与浓氨水、水合肼、氢氧化 锶、氢氧化钡和硫酸铜在一定条件下反应得到相应的盐， 并将其应用于气体发生剂和少烟彩色火焰烟火的研究. 5,5'-联四唑分子和它的阴离子 $\mathrm{BT}^{2-}$, 可以作为配位化合 物的单、双、三或者四配体. 2008 年 Eulgem 等 ${ }^{[32]}$ 以 $\mathrm{BT}^{2-}$ 和 3,6-双( $2 H$-四唑)-1,2,4,5-四嗪(BTT)的离子

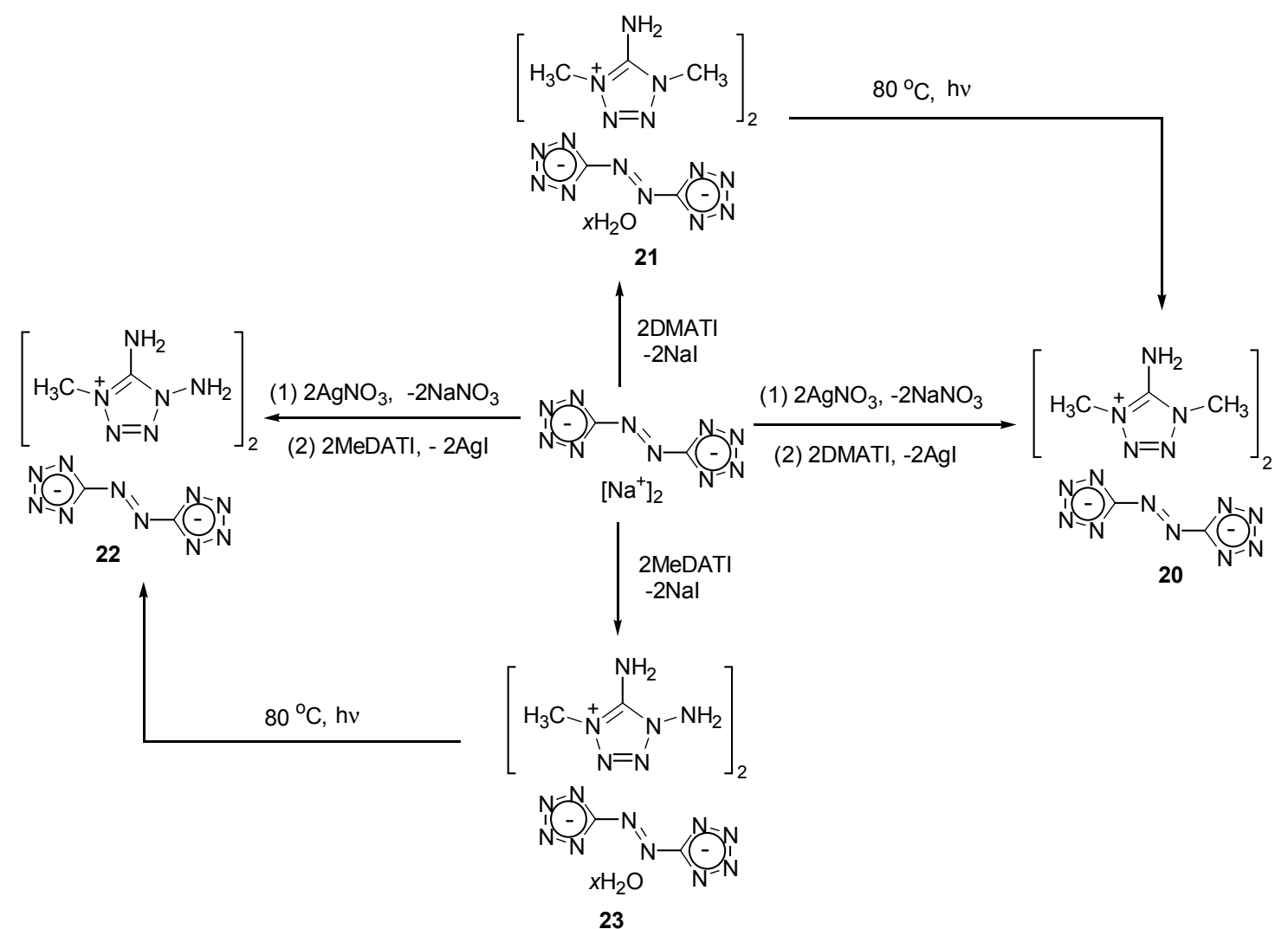

Scheme 8 


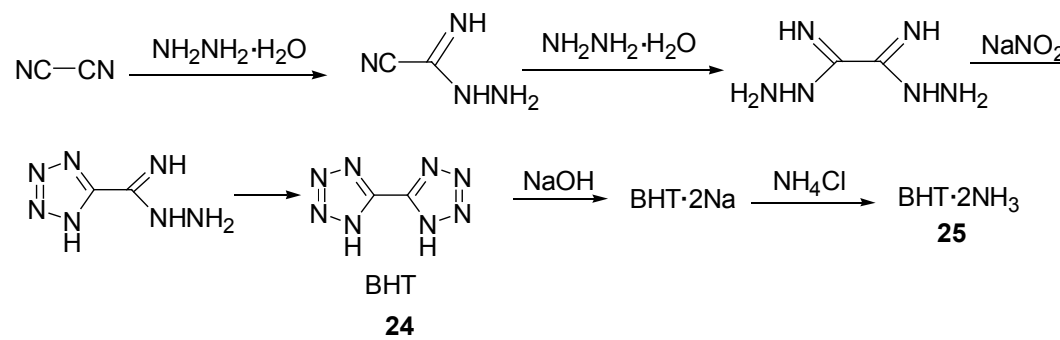

\section{Scheme 9}

$\mathrm{BTT}^{3-}$ 为阴离子, 得到新型的稀土金属配合物(Scheme 10). 联四唑锰盐(MnBT, 26) 是合成 $\mathbf{2 4 , 2 7}$ 和 28 的理想 前体. 由于这些碱土金属四唑化合物不溶于水和有机溶 剂, 对它们进行质谱或者红外检测较困难, 目前还没有 发现其在含能材料方面的应用.

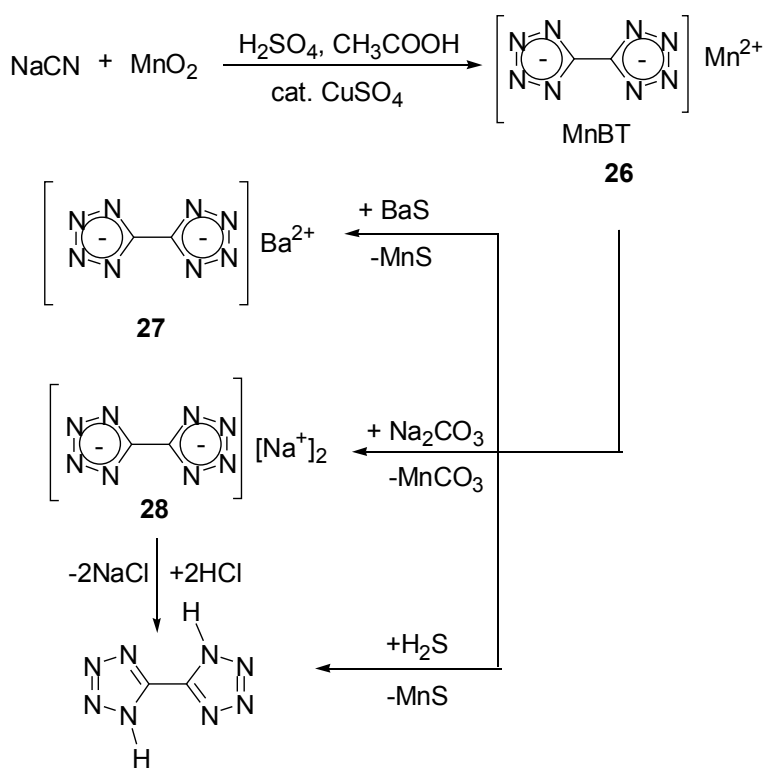

Scheme 10

\section{3 联氨四唑}

2008 年 Klapötke 等 ${ }^{[33]}$ 用 4 与过量的镁粉在水中反 应，经过酸化得到 9, 9 为白色粉末，产率提高到 95.2\%。 9 为钝感、高氮含量的化合物(含氮 83.3\%), 爆速 $(8523$ $\mathrm{m} / \mathrm{s}$ )、爆压 $(27.7 \mathrm{GPa})$, 分别与 RDX 和 PETN 接近, 而且 热稳定性很好 $\left(>200{ }^{\circ} \mathrm{C}\right)$. Eberspächer 等 ${ }^{[34]}$ 进一步研究 发现 9 分别可以与酸和碱反应, 作为阳离子或者阴离子 形成非金属含能盐(Scheme 11), 此方法有望应用到大 量的有机含能盐的合成上.

\section{4 联四唑胺}

2005 年 Friedrich 等 ${ }^{[35]}$ 以联四唑胺 BTA 为配体合成 出三个 BTA 的铜络合物. 文章给出了两种 $\mathrm{H}_{2} \mathrm{BTA} \cdot \mathrm{H}_{2} \mathrm{O}$ 的合成方法, 方法一: 双氧氨钠盐与叠氮钠在酸催化条 件下反应，高产率(89\%)地得到 $\mathrm{H}_{2} \mathrm{BTA} \cdot \mathrm{H}_{2} \mathrm{O}$; 方法二:

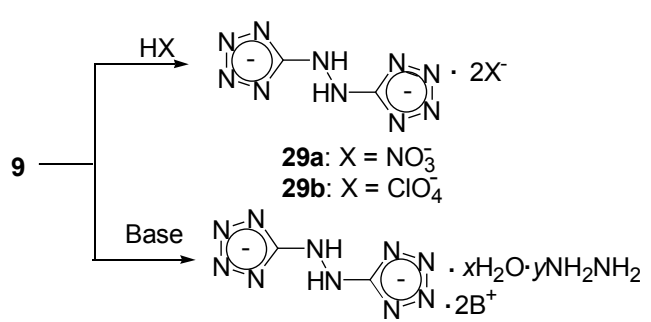

30a: $\mathrm{B}^{+}=\mathrm{NH}_{4}^{+}, x=2, y=2 ; \quad 30 \mathrm{~b}: \mathrm{B}^{+}=\mathrm{NH}_{2} \mathrm{NH}_{3}, x=0, y=0$ 30c: $\mathrm{B}^{+}=\mathrm{NH}_{2} \mathrm{NH}_{3}^{+}, x=0, y=1 ; \quad 30 \mathrm{~d}: \mathrm{B}^{+}=\left(\mathrm{NH}_{2}\right)_{3} \mathrm{C}^{+}, x=0, y=0$ 30e: $\mathrm{B}^{+}=\left(\mathrm{NH}_{2}\right)_{2}\left(\mathrm{NH}_{2} \mathrm{NH}\right) \mathrm{C}^{+}, x=0, y=0$

\section{Scheme 11}

采用澳化氰与氨基四唑一水合钠盐在氢氧化钠碱性条 件下反应得到目标产物. $\mathrm{H}_{2} \mathrm{BTA} \cdot \mathrm{H}_{2} \mathrm{O}$ 在 $120{ }^{\circ} \mathrm{C}$ 真空条 件下脱去一分子水, 得到 $\mathrm{H}_{2} \mathrm{BTA}$. $\mathrm{H}_{2} \mathrm{BTA}$ 和 $\mathrm{BTA}^{2-}$ 分别 在稀氨水中反应, 而 $\mathrm{HBTA}^{-}$是在浓氨水中反应(Scheme 12). $\mathrm{H}_{2} \mathrm{BTA}$ 和 $\mathrm{BTA}^{2-}$ 在稳定性、感度和能量方面均具有 良好性能，可以作为烟火的添加剂和以 AP 为基础的火 箭推进剂的组分.

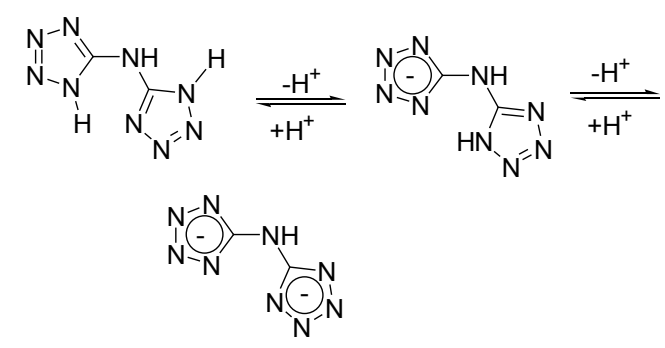

\section{Scheme 12}

Guo 等 ${ }^{[36]}$ 以 $\mathrm{BTA}^{2-}$ 为阴离子与有机阳离子反应高 产率(超过 95\%)的合成出 8 种四唑盐. 计算表明这 8 个 化合物的密度均超过 $1.5 \mathrm{~g} / \mathrm{cm}^{3}$, 生成热较大, 分解温度 超过 $160{ }^{\circ} \mathrm{C}$, 在室温下可以稳定存放几个月. 2010 年 Guo 等 ${ }^{[37]}$ 在此基础上成功合成出联四唑胺盐 $33 \mathbf{a} \sim 33 \mathbf{i}$ (Scheme 13), 并且对其做了充分的特性分析：所有产物 均为白色固体且产率较高，大部分产物产率超过 $95 \%$. 所有的化合物表现出了良好的物理性质，包括高密度 $\left(1.55 \sim 1.75 \mathrm{~g} / \mathrm{cm}^{3}\right.$ )、良好的热稳定性(分解温度为 $189 \sim$ $\left.169{ }^{\circ} \mathrm{C}\right)$. 这些高能材料爆速计算值 $(7677 \sim 9487 \mathrm{~m} / \mathrm{s})$ 、 

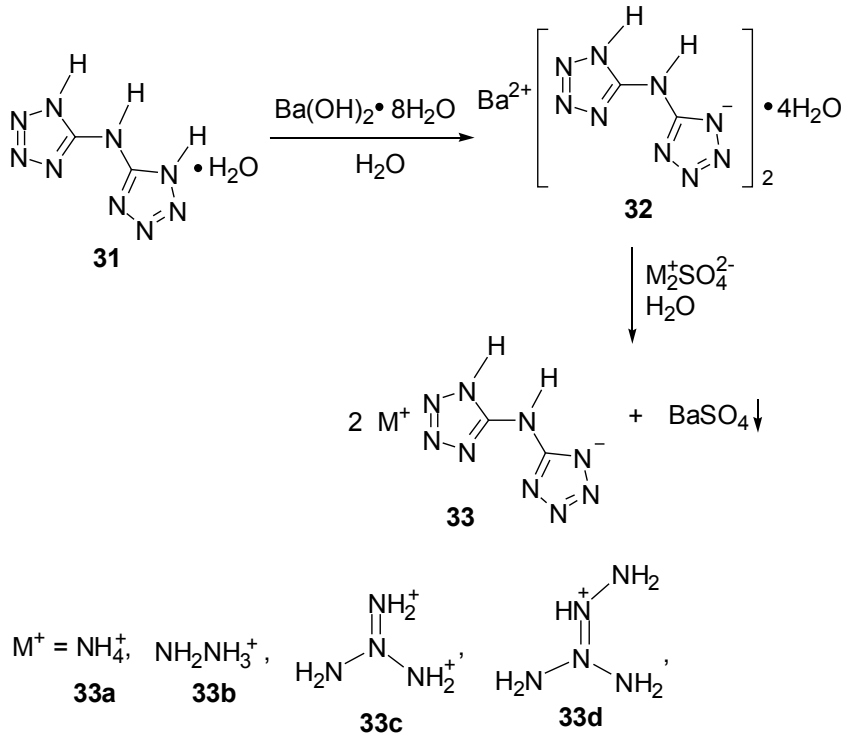

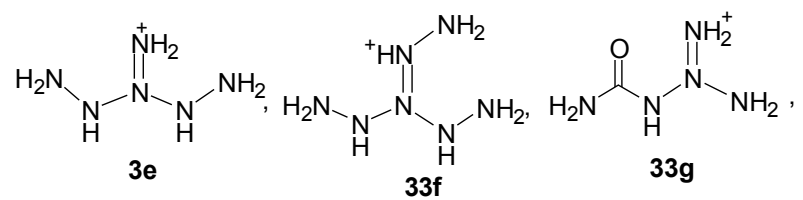<smiles>Cn1nnnc1N</smiles>

$33 \mathrm{~h} \quad 33 \mathrm{i}$

Scheme 13

爆压 $(19.4 \sim 33.6 \mathrm{~kJ} / \mathrm{mol})$, 与 TNT, RDX 和 HMX 相当, 有望成为一种新型的高性能炸药.

\section{5 硝胺基双环四唑}

硝胺基四唑化合物具有高氮含量、芳香性、热稳定 性好等性质. 硝胺基四坐系列化合物可以作为新型含能
材料, 特别在低毒含能材料方面很有用处.

2010 年 Joo 等 ${ }^{[38,39]}$ 连续发表了两篇关于硝胺基四唑 合成的文章. 反应过程为: 叠氮氰与甲氧基氨反应生成 1-甲氧基-5-氨基四唑，室温条件下用 100\%的硝酸硝化 氨基四唑得到 1-甲氧基-5-硝胺基四唑，在此基础上很 容易得到硝胺基四唑盐(Scheme 14). 产物具有良好的 稳定性、高密度、强吸热性、高爆压和高爆速，与 HMX 相近 $(P=39.63 \mathrm{GPa}, D=9320 \mathrm{~m} / \mathrm{s})$, 但是它们的撞击感 度比较高 $(1$ 和 $1.5 \mathrm{~J})$, 通过与低感度的氧化剂(比如硝酸 铵)结合可以降低感度.

\section{6 硝基双环四唑}

1997 年 Koldobskii 等 ${ }^{[40]}$ 对硝基取代的双环四唑化 合物进行了深入研究. 以 5-硝基四唑钠盐四水合物为起 始原料合成出 2,5-二取代四唑衍生物 39 和 40 (Scheme 15). 产物含氮量较高, 合成方法简单, 产率较高, 在空 气中稳定，但是目前对各方面的性质研究不是很充分. 5-硝基四唑钠的水合物为原料有望合成出更多的 5-硝基 四唑类含能化合物.

5-硝基- $2 H$-四唑的氢酸性较强，易成盐. 2009 年 Klapötke 等 ${ }^{[41]}$ 用 5-硝基四唑铵盐与氯代乙腈反应，氧基 与叠氮化钠成环反应生成 41,41 成盐得到一类新型的含 能材料 42 44 (Scheme 16). 化合物 41b 是性能良好、 高吸热的富氮盐，与普通的含能材料相比感度低，可以 作为钝感、环境友好的含能材料，有良好的应用前景.

\section{7 烷基相连的双环四唑}

2002 年 Bronisz ${ }^{[42]}$ 报道了一种新型的双四唑环化合 物—-2-取代四唑化合物 $\mathbf{4 5} .45$ 只能通过 $1 H-2,3,4$-四唑 的烷基化来获得, 乙腈重结晶后得到无色的结晶, 熔点 为 $115 \sim 116{ }^{\circ} \mathrm{C}$, 热力学分析表明, 化合物 EBTZ (45)在

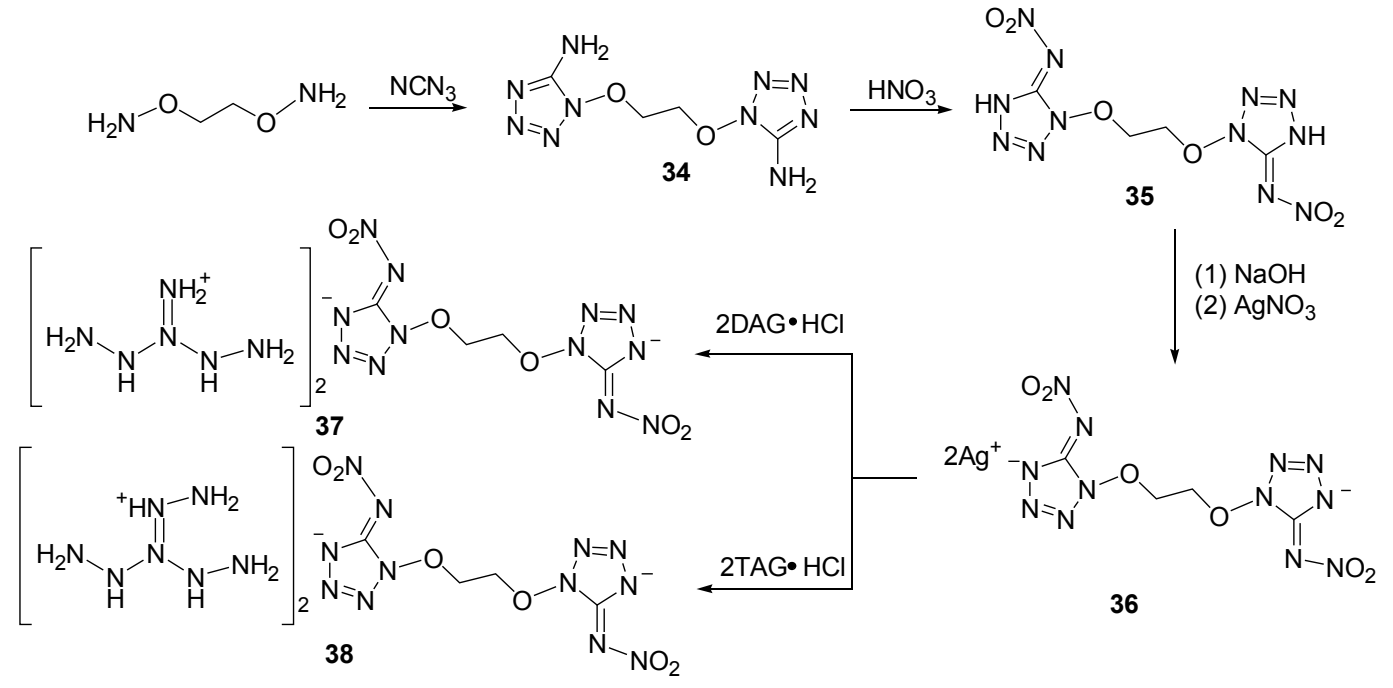

Scheme 14 


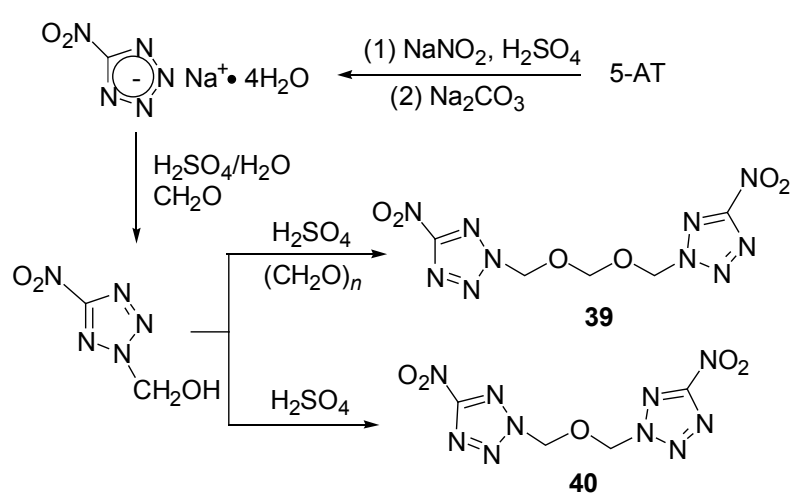

Scheme 15

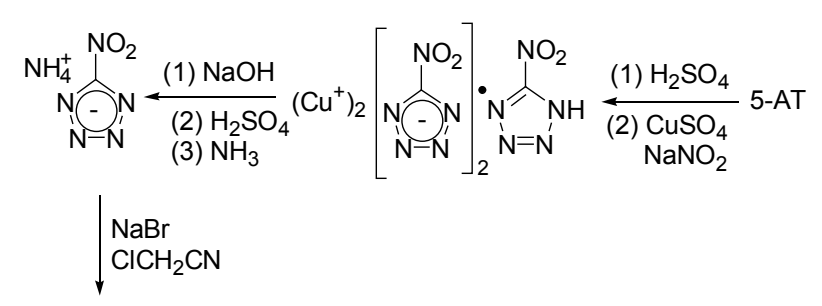

$$
\text { (1) } \mathrm{H}^{+} \mathrm{H}_{4} \mathrm{Cl} \text { (aq.) }
$$$$
\text { (- }
$$$$
\mathrm{hv}, \triangle \longrightarrow \text { 41a: } x=1
$$$$
\begin{aligned}
{[\text { Cat }]^{+}=} & \mathrm{NH}_{4}^{+}(\mathbf{4 2 a}: x=1) \\
& \mathrm{NH}_{4}^{+}(\mathbf{4 2} \mathbf{b}: x=0) \\
& \left(\mathrm{NH}_{2}\right)_{3} \mathrm{C}^{+}(\mathbf{4 3}: x=0) \\
& \left(\mathrm{NH}_{2} \mathrm{NH}\right)\left(\mathrm{NH}_{2}\right)_{2} \mathrm{C}^{+}(44: x=0)
\end{aligned}
$$

\section{Scheme 16}

达到 $200{ }^{\circ} \mathrm{C}$ 以上时就会分解. 45 与 $\mathrm{Zn}\left(\mathrm{ClO}_{4}\right)_{2} \cdot 6 \mathrm{H}_{2} \mathrm{O}$ 以 $1: 3$ 的比例在乙腈(含水 $1 \%$ )中反应得到的络合物为 $\left\{\left[\mathrm{Zn}(\mathrm{EBTZ})_{3}\right]\left(\mathrm{ClO}_{4}\right)_{2}\right\}_{n}$. 产物在低温乙腈、甲醇中不溶, 但是溶解度随着温度的升高而增加. 目前对 2-取代四唑 含能化合物的研究还不是很深入, 对其各方面的性质了 解还不够.

$$
\stackrel{N=N}{N}{ }_{N^{\prime}}^{N} N_{45}^{N=}=
$$

2003 年, Bottaro 等 ${ }^{[43]}$ 在 SRI International 上发表文 章, 介绍了一些双环四唑的合成路线, 可以为高能材料 的合成提供一个平台. 双氰化合物与叠氮有机盐反应得 到双四唑化合物 46 (Scheme 17). 反应物氰化物和叠氮 盐都有很大的毒性, 使该合成方法应用受限, 需要作进 一步的改进.

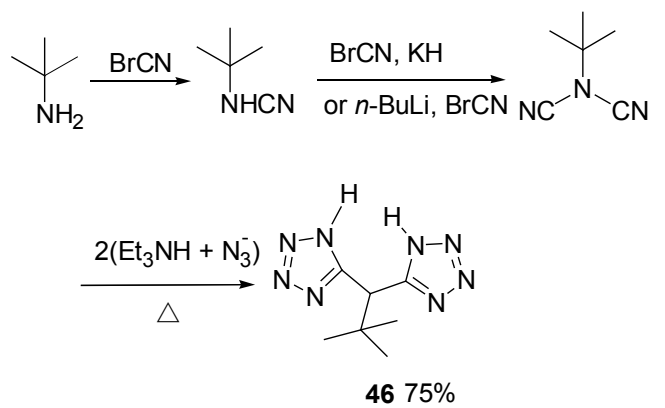

Scheme 17

\section{8 其它双环四唑}

Löbbecke 等 ${ }^{[44]}$ 采用 DSC, TGA, EGA 等分析方法, 研究了约 60 种含有四唑环或者四嗪环的富氮化合物热 力学性质. 含有双四唑环的联四唑化合物的分解放出的 热量远大于单环四唑化合物, 当增加一个四唑环之后化 合物分解时所放出的热量远大于其二倍, 其分解温度较 高, 感度也较低, 与传统的含能材料相比优势明显.

2001 年 Sauer 等 ${ }^{[14]}$ 采用 1915 年 Curtius 的合成方法 合成出了 BTT (48), 48 自 $231{ }^{\circ} \mathrm{C}$ 开始剧烈分解至 $236{ }^{\circ} \mathrm{C}$ 分解结束. 分解热达到 $533 \mathrm{~kJ} / \mathrm{mol}$, 大约是 TNT 的四倍. 48 可以发生 Diels-Alder 反应，生成目标产物 49 和 50 (Scheme 18).
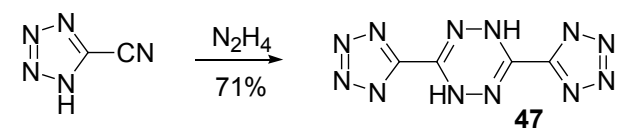

$\mathrm{CrO}_{3} \downarrow 72 \%$

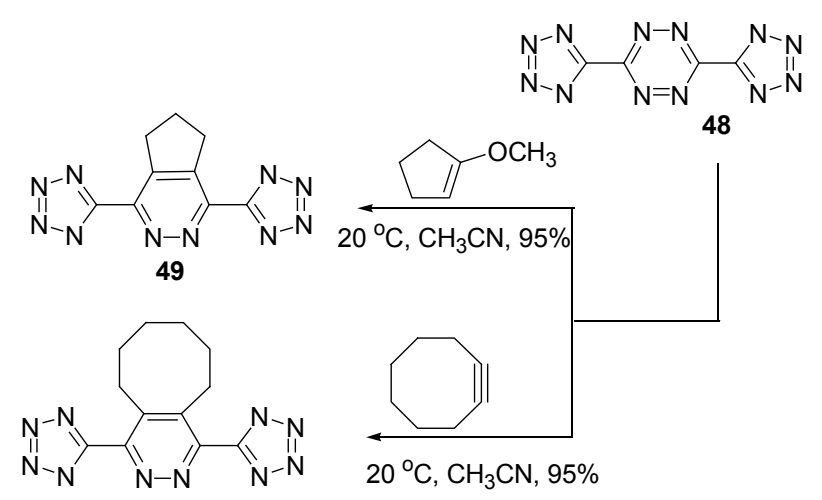

50

\section{Scheme 18}

低爆热富氮化合物的发展引起了人们很大的兴趣. 2009 年 Heppekausen 等 ${ }^{[45]}$ 从很简单的伯氨出发制得肼 基四唑, 然后在溴和乙酸的作用下得到目标产物双四唑 基四氮烯 51 (Scheme 19), 其爆热较低, 有可能用作汽 车安全气囊. 文章给出了高效生产四唑氮烯的一般方 法, 以及一个新的高产率合成甲基四唑基肼的方法. 


$$
\underset{\text { Sodium azide, acetic acid }}{\stackrel{\text { Monomethyl hydrazine }}{\text { (2 equiv.) }}}
$$$$
\stackrel{\text { Bcetic acid, water }}{\text { B. }}
$$

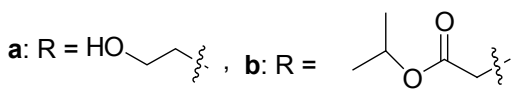

\section{Scheme 19}

2008 年 Joo 等 ${ }^{[46]}$ 通过改变现有的实验路线制得 1取代-5-氨基联四唑. 二胺化合物与 5 6 倍物质的量的 溴化氯以及过量的叠氮钠反应, 得到联四唑化合物. 采 用同样的路线用三氨乙基三氨与叠氮氰在水/乙腈中反 应，得到三取代氨基四唑.

\section{2 多环四唑}

随着研究的不断深入, 一些更复杂的多环四唑被合 成出来, 其可以作为气体发生剂和下一代火箭推进剂系 统的燃速调节剂以及生物材料等. 2008 年 Chafin 等 ${ }^{[47]}$ 把着乙基引入四唑化合物, 得到羟乙基双四唑化合物 54 和 57 (Scheme 20). 其中羟乙基四唑极性较大, 柱色 谱分离时用常用的溶剂难以洗脱, 但是用甲醇和丙酮或 者乙酸乙酯会把产物洗脱下来, 产物里面会含有氯化 钠, 而正丙醇会溶解产物但是不溶解盐, 通过这个方法 得到了分析纯的差隹乙基四唑化合物.

2009 年 Touti 等 ${ }^{[48]}$ 合成出了高度对称的含有 4 个四 唑基团的二乙胺化合物 58 59 (Scheme 21), 得到纯 58 产率为 $82 \%$, 纯 59 产率为 $70 \%$. 合成方法简单, 有望应 用到其它多环四唑含能化合物的合成.

2006 年 Bond 等 ${ }^{[49]}$ 报道了长链卤代烷取代的双四 唑. 以 1,4-二(四唑基)衍生物为起始原料与二溴化合物 反应得到相应的目标产物 60 , 并对 60 进行了核磁分析 和 $\mathrm{X}$ 射线分析(Scheme 22).

2007 年 Bond 等 ${ }^{[50]}$ 合成和表征了大环四唑化合物. 在固相里, 大环化合物的构型受到苯环上面四唑环定 位、烷基链长以及分子间作用的影响. 该小组已经报道 了一些用四唑环作为配体与这些金属或者碱土金属反 应形成络合物的研究成果.

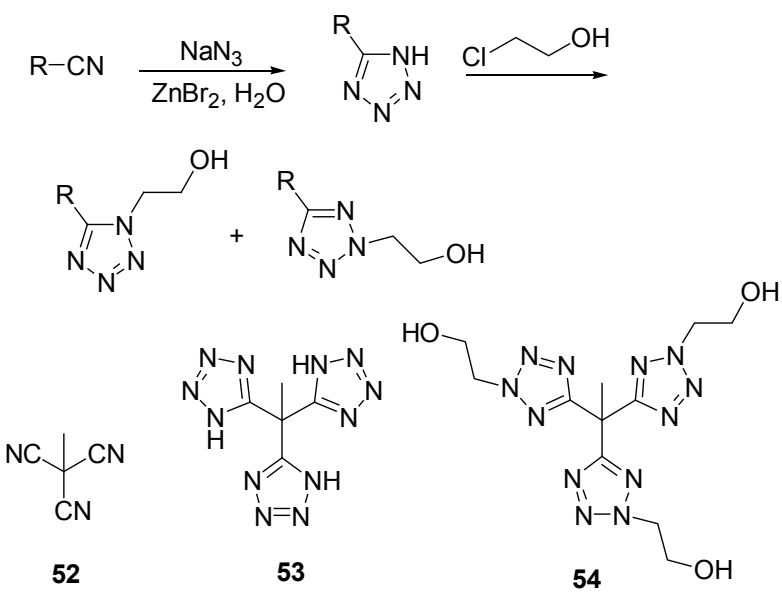<smiles>N#CC(C#N)CC(C#N)C#N</smiles><smiles>OCCn1nnc(C(CC(c2nnn(CCO)n2)c2nnn(CCO)n2)c2nnn(CCO)n2)n1</smiles>

Scheme 20<smiles>NCCNCc1nnnn1Cc1nnnn1Cc1nnnn1Cc1nnnn1Cc1ccccc1</smiles>

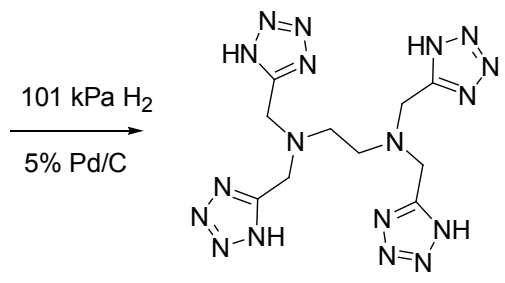

59

Scheme 21

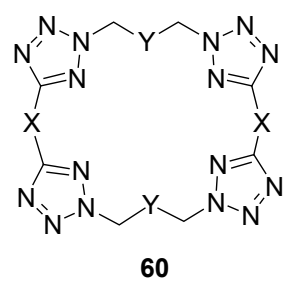

Scheme 22 
应用较广的聚乙烯四唑含有大量的四唑环, 因其具 有强大的能量、高的热力学稳定性, 在很多方面都有广 泛的应用，包括炸药、特种聚合物等. 2004 年 Huang

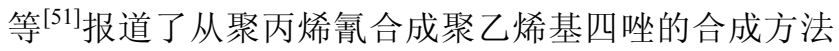
(Eq. 1). 以 DMF 为溶剂, 用便宜的聚丙烯氭(PAN)作为 起始原料与叠氮钠在 $60 \sim 120{ }^{\circ} \mathrm{C}$ 下反应制得 PVTs, 四 唑环的含量从 $18.3 \% \sim 97.5 \%$ 不等. 得到的四唑化产率 最高为 $98 \%$, 固有粘度达到 $10.5 \mathrm{dL} / \mathrm{g}$, 四唑含量为 97.5\%. 四唑含量比较高的 PVTs 在爆炸时放出大量的 热, 可以作为火箭推进剂的一个有效组分.

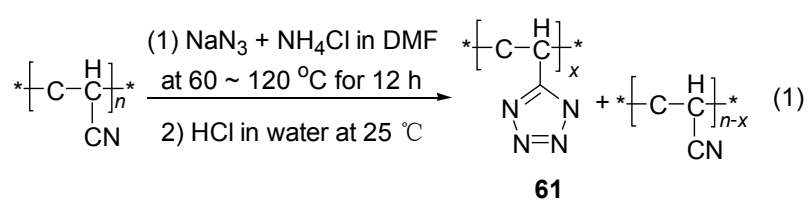

2010 年俄罗斯科学家 Vereshchagin 等 $^{[52]}$ 以三氯三 嗪为原料与四唑化合物反应制得三取代的四唑含能化 合物. 丙酮和水为溶剂, 加入三乙胺或者 $\mathrm{NaHCO}_{3}$ 反应 得到对应的四唑化合 $62 \mathrm{a} \sim 62 \mathrm{c}(\mathrm{Eq} .2)$. 三氯三嗪可以 作为亲核取代反应的底物与不同的四唑化合物合成得 到稳定的产物. 但目前对于多环四唑化合物的爆轰性 能、感度、密度等特性还没有系统的研究报道.
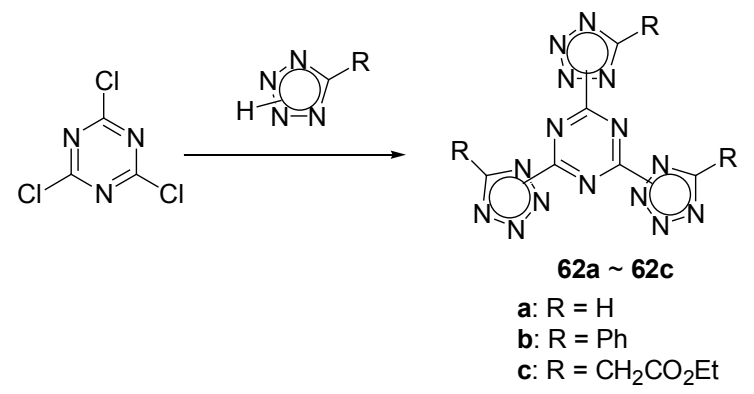

\section{3 结语与展望}

双环和多环四唑化合物因含大量的四唑环、高氮含 量、产气多为 $\mathrm{N}_{2}$ 等一系列优良性质, 有望在新型炸药、 火箭推进剂、气体发生剂、低烟或无烟烟火方面应用而 得到了广泛关注, 近年来大量新型双环或多环四唑化合 物被合成出来, 针对其中一些性能良好的化合物已经在 开展应用研究.

下一步双环和多环四唑化合物的研究重点仍集中 在两个方面: (1)与单环四唑相比, 双环和多环四唑化合 物修饰位点更多, 可以引入更多的含能基团, 应系统研 究其构效关系, 设计合成综合性能更好的新型含能材 料, 在提高密度、爆轰、爆压、爆速等爆轰性能的同时 降低感度, 增强安定性. (2)由于合成路线长, 使用原料、 中间体很多毒性比较大等因素, 双环和多环四唑化合物
的制备成本还比较高，限制了其应用和推广，应开发高 效、安全的新合成反应，降低生产成本.

综上所述，双环和多环四唑含能化合物表现出了很 大的发展潜力, 相信随着科学的发展, 四唑环类化合物 在含能材料的应用领域必将更加广阔.

\section{References}

[1] Chavez, D. E.; Hiskey, M. A.; Gilardi, R. D. Angew. Chem., Int. Ed. 2000, 39, 1791.

[2] Xue, H.; Gao, H. X.; Twamley, B.; Shreeve, J. M. Eur. J. Inorg. Chem. 2006, 2959.

[3] Rossi, R. A.; Pierini, A. B.; Peñéñory, A. B. Chem. Rev. 2003, 103, 71.

[4] Bruney, L. Y.; Bledson, T. M.; Strout, D. L. Inorg. Chem. 2003, 42, 8117.

[5] Srinivasan, B. R.; Girkar, S. V.; Raghavaiah, P. Acta Crystallogr. 2007, E63, $\mathrm{m} 3100$.

[6] Himo, F.; Demko, Z. P.; Noodleman, L.; Sharpless, K. B. J. Am. Chem. Soc. 2003, 125, 9983.

[7] Li, G. Q.; Li, Y. C.; Ma, Q. L.; Sun, C. H.; Pang, S. P. Chin. J. Chem. 2010, 30, 1431 (in Chinese). (李冠琼, 李玉川, 马巧丽, 孙成辉, 庞思平, 有机化学, 2010, 30,1431 .)

[8] Thiele, J. Justus Liebigs Ann. Chem. 1892, 270, 54.

[9] Sivabalan, R.; Talawar, M.; Senthilkumar, N.; Kavitha, B.; Asthana, S. N. J. Therm. Anal. Calorim. 2004, 78, 781.

[10] Hiskey, M. A.; Goldman, N.; Stine, J. R. J. Energ. Mater. 1998, 16, 119.

[11] Hammerl, A.; Klapötke, T. M.; Nöth, H.; Warchhold, M.; Holl, G.; Kaiser, M.; Ticmanis, U. Inorg. Chem. 2001, 40, 3570.

[12] Serebryanskaya, T. V.; Matulis, V. E.; Lyakhov, A. S.; Voitekhovich, S. V.; Gaponik, P. N.; Ivashkevich, O. A. Heteroat. Chem. 2010, 21, 24.

[13] Gao, H. X.; Ye, C. F.; Piekarski, C. M.; Shreeve, J. M. J. Phys. Chem. C 2007, 111, 10718 .

[14] Sauer, J.; Pabst, G. R.; Holland, U.; Kim, H. S.; Loebbecke, S. Eur. J. Org. Chem. 2001, 697.

[15] Hammerl, A.; Hiskey, M. A.; Holl, G.; Klapötke, T. M.; Polborn, K.; Stierstorfer, J.; Weigand, J. J. Chem. Mater. 2005, 17, 3784.

[16] Hammerl, A.; Holl, G.; Klapötke, T. M.; Mayer, P.; Nöth, H.; Piotrowski, H.; Warchhold, M. Eur. J. Inorg. Chem. 2002. 834.

[17] Singh, G.; Prajapati, R.; Frohlich, R. J. Hazard. Mater. 2005, A118, 75.

[18] Klapötke, T. M.; Sabaté, C. M. Z. Anorg. Allg. Chem. 2007, 633, 2671.

[19] Tao, G. H.; Twamley, B.; Shreeve, J. M. Inorg. Chem. 2009, 48, 9918.

[20] Xu, S. L.; Yang, S. Q.; Yue, S. T. Chin. J. Explos. Propellants 2005, 28, 52 (in Chinese).

(徐松林，阳世清，岳守体，火炸药学报， 2005, 28, 52.)

[21] Sivabalan, R.; Anniyappan, M.; Pawar, S. J.; Talawar, M. B.; Gore, G. M.; Venugopalan, S.; Gandhe, B. R. J. Hazard. Mater. 2006, A137, 672 .

[22] Wang, Y. H.; Du, Z. M.; He, C. L.; Cong, X. M.; Wang, H. S.; Wang, C. Y. Chin. J. Energ. Mater. 2008, 16, 581(in Chinese). (王义惠, 杜志明, 何春林, 丛晓民, 王宏社, 王春迎, 含能材料, 2008, 16, 581.)

[23] Xu, S. L.; Yang, S. Q.; Yue, S. T. Chin. J. Synth. Chem. 2005, 13, 486 (in Chinese). 
(徐松林，阳世清，岳守体，合成化学, 2005, 13,486.)

[24] Tappan, B. C.; Ali, A. N.; Son, S. F. Propellants, Explos., Pyrotech. 2006, 31, 163.

[25] Tremblay, M. Can. J. Chem. 1965, 43, 1230.

[26] Hiskey, M. A.; Goldman, N.; Stine, J. R. J. Energ. Mater. 1998, 16, 119.

[27] Ye, C. F.; Xiao, J. C.; Twamley, B.; Shreeve, J. M. Chem. Commun. 2005, 2750

[28] Klapötke, T. M.; Sabaté, C. M. Chem. Mater. 2008, 20, 1750.

[29] Gazz, E. O. Chim. Ital. 1920, 50, 256.

[30] Hyoda, S.; Kita, M.; Sawada, H.; Nemugaki, S.; Otsuka, S.; Miyawaki, Y.; Ogawa, T.; Kubo, Y. US 6040453, 2000 [Chem. Abstr. 2000, 133, 225201].

[31] Hiskey, M. A.; Chavez, D. E.; Naud, D. L. US 6214139, 2001 [Chem. Abstr. 2001, 134, 282929].

[32] Eulgem, P. J.; Klein, A.; Maggiarosa, N.; Naumann, D.; Pohl, R. W. H. Chem. Eur. J. 2008, 14, 3727.

[33] Klapötke, T. M.; Sabaté, C. M. Chem. Mater. 2008, 20, 3629.

[34] Eberspächer, M.; Klapötke, T. M.; Sabaté, C. M. Helv. Chim. Acta 2009, 92, 977 .

[35] Friedrich, M.; Gálvez-Ruiz, J. C.; Klapötke, T. M.; Mayer, P.; Weber, B.; Weigand, J. J. Inorg. Chem. 2005, 44, 8044.

[36] Guo, Y.; Gao, H. X.; Twamley, B.; Shreeve, J. M. Adv. Mater. 2007, 19, 2884.

[37] Guo, Y.; Tao, G. H.; Zeng, Z.; Gao, H. X.; Parrish, D. A.; Shreeve, J. M. Chem. Eur. J. 2010, 16, 3753.
[38] Joo, Y. H.; Shreeve, J. M. Angew. Chem., Int. Ed. 2010, 49, 7320

[39] Joo, Y. H.; Shreeve, J. M. J. Am. Chem. Soc. 2010, 132, 15081.

[40] Koldobskii, G. I.; Soldatenko, D. S.; Gerasimova, E. S.; Khokhryakova, N. R.; Shcherbinin, M. B.; Lebedev, V. P.; Ostrovskii, V. A. Russ. J. Org. Chem. 1997, 33, 1771.

[41] Klapötke, T. M.; Sabaté, C. M.; Rasp, M. J. Mater. Chem. 2009, 19, 2240.

[42] Bronisz, R. Inorg. Chim. Acta 2002, 340, 215.

[43] Bottaro, J. C.; Petrie, M.; Penwell, P. E.; Dodge, A. L.; Malhotra, R. SRI International 2003

[44] Löbbecke, S.; Pfeil, A.; Krause, H. H. Propellants, Explos., Pyrotech. 1999, 24, 168.

[45] Heppekausen, J.; Klapötke, T. M.; Sproll, S. M. J. Org. Chem. 2009, 74, 2460.

[46] Joo, Y. H.; Shreeve, J. M. Org. Lett. 2008, 10, 4665.

[47] Chafin, A.; Irvin, D. J.; Mason, M. H.; Mason, S. L. Tetrahedron Lett. 2008, 49, 3823.

[48] Touti, F.; Maurin, P.; Hasserodt, J. Eur. J. Org. Chem. 2009, 1495.

[49] Bond, A. D.; Fleming, A.; Kelleher, F.; McGinley, J.; Prajapati, V. Tetrahedron 2006, 62, 9577.

[50] Bond, A. D.; Fleming, A.; Kelleher, F.; McGinley, J.; Prajapati, V.; Skovsgaard, S. Tetrahedron 2007, 63, 6835.

[51] Huang, M. R.; Li, X. G.; Li, S. X.; Zhang, W. React. Funct. Polym. 2004, 59, 53.

[52] Vereshchagin, L. I.; Verkhozina, O. N.; Pokatilov, F. A.; Proidakov, A. G.; Kizhnyaev, V. N. Chem. Heterocycl. Compd. 2010, 46, 206.

(Qin, X.) 